\title{
Overtone resonance enhanced single-tube on-beam quartz enhanced photoacoustic spectrophone
}

\author{
Huadan Zheng, ${ }^{1,2}$ Lei Dong, ${ }^{1,2, a)}$ Angelo Sampaolo, ${ }^{3,4}$ Pietro Patimisco, ${ }^{3,4}$ Weiguang Ma, ${ }^{1,2}$ \\ Lei Zhang, ${ }^{1,2}$ Wangbao Yin, ${ }^{1,2}$ Liantuan Xiao, ${ }^{1,2}$ Vincenzo Spagnolo, ${ }^{3}$ Suotang Jia, ${ }^{1,2}$ \\ and Frank K. Tittel ${ }^{4}$ \\ ${ }^{1}$ State Key Laboratory of Quantum Optics and Quantum Optics Devices, Institute of Laser Spectroscopy, \\ Shanxi University, Taiyuan 030006, China \\ ${ }^{2}$ Collaborative Innovation Center of Extreme Optics, Shanxi University, Taiyuan 030006, China \\ ${ }^{3}$ Dipartimento Interateneo di Fisica, Università degli Studi di Bari and Politecnico di Bari, CNR-IFN BARI, \\ Via Amendola 173, Bari 70126, Italy \\ ${ }^{4}$ Department of Electrical and Computer Engineering, Rice University, Houston, Texas 77005, USA
}

(Received 14 July 2016; accepted 1 September 2016; published online 14 September 2016)

\begin{abstract}
A single-tube on-beam quartz enhanced photoacoustic spectroscopy (SO-QEPAS) spectrophone, which employs a custom-made quartz tuning fork (QTF) having a prong spacing of $700 \mu \mathrm{m}$ and operating at the 1st overtone flexural mode, is reported. The design of QTF prong geometry allows the bare QTF to possess twice higher $Q$-factor values for the 1 st overtone resonance mode falling at $\sim 17.7 \mathrm{kHz}$ than in the fundamental resonance mode at $\sim 2.8 \mathrm{kHz}$, resulting in an 8 times higher QEPAS signal amplitude when operating in the 1 st overtone resonance mode. Both the vertical position and length of the single-tube acoustic micro-resonator (AmR) were optimized to attain optimal spectrophone performance. Benefiting from the high overtone resonance frequency and the quasi 1st harmonic acoustic standing waves generated in the SO-QEPAS configuration, the AmR length is reduced to $14.5 \mathrm{~mm}$. This allows the realization of compact spectrophone and facilitates the laser beam alignment through the QTF + AmR system. The signal enhancement in the overtone resonance mode and the high acoustic coupling efficiency between the AmR and QTF in the SOQEPAS configuration yields an overall sensitivity enhancement factor of $\sim 380$ with respect to the bare custom QTF operating in the fundamental resonance mode. Published by AIP Publishing.

[http://dx.doi.org/10.1063/1.4962810]
\end{abstract}

Quartz enhanced photoacoustic spectroscopy (QEPAS), an alternative approach to photoacoustic spectroscopy (PAS) utilizing a quartz tuning fork (QTF) as a sharply resonant acoustic transducer instead of microphone, ${ }^{1-6}$ has been applied to environmental monitoring, industrial process control, and medical diagnosis. ${ }^{7-22}$ It combines PAS's inherent merits of zero-background and excitation light wavelength independence with the QTF's characteristics of environmental noise immunity, providing a robust, ultra-compact, and cost-effective spectrophone for trace gas detection. ${ }^{3,23,24}$

A conventional QEPAS spectrophone employs a commercial QTF having a resonance frequency of $\sim 32 \mathrm{kHz}$, a prong spacing of $\sim 300 \mu \mathrm{m}$, and an acoustic micro-resonator (AmR) to confine the sound wave and enhance the QEPAS signal. ${ }^{25}$ The typical AmR consists of two thin tubes aligned perpendicularly to the QTF plane and previous studies showed that the optimum AmR length is between $\lambda_{\mathrm{s}}$ and $\lambda_{\mathrm{s}} / 2$, where $\lambda_{\mathrm{s}}$ is the sound wave wavelength at the QTF resonance frequency. For the commercial QTF, the optimal AmR total length is $\sim 9 \mathrm{~mm}^{25}$ It is critical that the laser beam, which enters the AmR, does not hit the tubes walls or the QTF internal surface in order to avoid photothermal effects.

QEPAS spectrophones based on commercial QTFs show good performance in the near-infrared (NIR) region, where distributed feedback lasers (DFBs) or external-cavity diode lasers (ECDLs) with good beam quality are typically

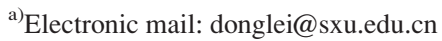

employed. ${ }^{7,14,20}$ However, when lasers with poor beam quality or large divergence are used, such as light emitting diodes (LEDs), stripe lasers, or mid-infrared (MIR) quantum cascade lasers (QCLs), the $300 \mu \mathrm{m}$ prong spacing can partly block the excitation light and produce an undesirable, nonzero fringe-like background, which can strongly limit the QEPAS sensitivity. ${ }^{9,21,26}$ In addition, the narrow prong spacing prevents the extension of the QEPAS technique in the terahertz $(\mathrm{THz})$ spectral region, due to the fact that in this range the wavelength is up to two orders of magnitude larger than that of a NIR spectral region and laser beam waist diameters smaller than 400-500 $\mu \mathrm{m}$ are not achievable for highly divergent $\mathrm{THz}$ quantum cascade laser beams. To overcome these limitations, custom QTFs with prong spacing in the range of 700-1000 $\mu \mathrm{m}$ have been designed and implemented in QEPAS sensors. ${ }^{11,27}$ For example, a custom QTF with a resonance frequency as low as $4.2 \mathrm{kHz}$ and $\sim 1 \mathrm{~mm}$ prong spacing has been implemented to provide the first demonstration of THz QEPAS sensor system, as reported in Ref. 11. Such a low resonance frequency resulted in an $81 \mathrm{~mm}-$ long acoustic wavelength, preventing the use of an AmR due to the difficulty in performing optical alignment. A sub-ppm detection level $\mathrm{H}_{2} \mathrm{~S}$ QEPAS sensor operating in the NIR range and based on a custom QTF with an $800 \mu \mathrm{m}$ prong spacing and a resonance frequency of $7.2 \mathrm{kHz}$ has been also demonstrated. ${ }^{10}$ In this case, two stainless steel tubes with a 1.3-mm inner diameter acting as an AmR were positioned on each side of the QTF, allowing to reach a $40 \times$ detection 
(a)

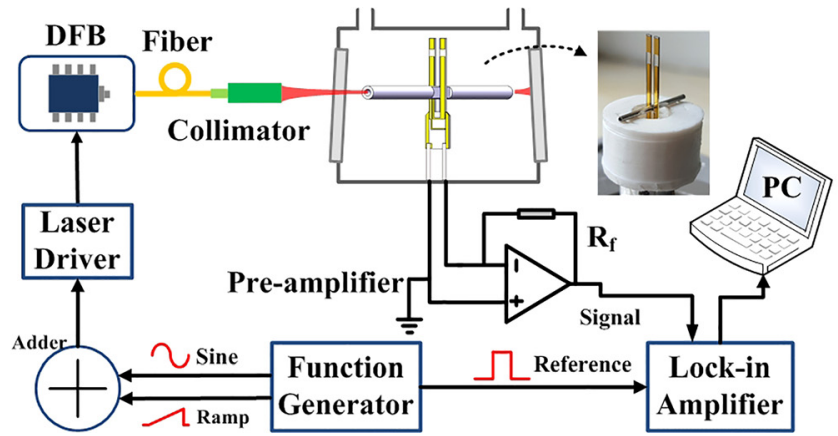

(b)

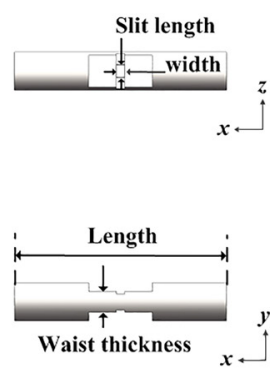

FIG. 1. Schematic diagram of the experimental setup (a) and the SOQEPAS AmR (b). DFB: distributed feedback laser; PC: personal computer; $\mathrm{R}_{\mathrm{f}}$ : feedback resistance. sensitivity enhancement factor. Nevertheless, the required AmR total length was $\sim 46 \mathrm{~mm}$, challenging for both laser beam alignment and spectrophone assembly. In a recent study, a single-tube AmR configuration was demonstrated to provide increased sensitivity enhancement factors and a reduction of the required AmR length. ${ }^{29}$ Due to the large prong spacing of the $7.2 \mathrm{kHz}$ custom QTF, a single-tube AmR was allowed to insert between the prongs, instead of positioning two independent tubes on each side of the QTF. The quasi standard acoustic standing waves generated in AmR reduced the optimal AmR length down to $38 \mathrm{~mm}$, and a sensitivity enhancement factor of $\sim 100$ was achieved, with respect to that obtained using a bare custom QTF. However, the 38-mm AmR length still remains $\sim 4$ times larger than the AmR used for the commercial $32 \mathrm{kHz}$ QTF.

Lowering the QTF fundamental resonance frequency reduces also the overtone frequencies, opening the way to their implementation in QEPAS sensor systems. ${ }^{4,5,28}$ By optimizing the QTF prongs sizes, overtone modes can provide a higher QEPAS sensor performance with respect to using the fundamental mode. ${ }^{5}$ Since the 1 st overtone frequency is $\sim 6.2 \times$ higher than the fundamental one, the AmR optical length can be significantly reduced. In this letter, we report a compact and highly sensitive QEPAS spectrophone employing a single-tube on-beam (SO-QEPAS) AmR configuration coupled with a custom QTF operating in the 1st overtone flexural mode at a frequency of $\sim 17.7 \mathrm{kHz}$.

The schematic diagram of the experimental setup is depicted in Fig. 1(a). Water vapor in the atmosphere was selected as the target analyte. A single-mode continuouswave distributed feedback (DFB) laser emitting at $1.37 \mu \mathrm{m}$ was employed as the excitation source to generate photoacoustic signals. The wavelength of the laser was scanned across the selected water absorption line by applying a voltage ramp wave to the laser current driver. In addition, the laser current was sinusoidally modulated at $f / 2$ by a function generator, where $f$ is the selected QTF resonance frequency. The laser beam was collimated through the QEPAS spectrophone by a fiber-coupled collimator (OZ optics Ltd. Model LPC-01), which produced a laser beam diameter of $\sim 200 \mu \mathrm{m}$ with a divergence angle of $9 \mathrm{mrad}$ on the QTF plane. The piezoelectric signal from the QTF was amplified by a custom transimpedance amplifier with a $10 \mathrm{M} \Omega$ feedback resistance and then processed by a lock-in amplifier (LIA) (Stanford Research Systems, Model SR830) to demodulate the signal in the $2 f$ mode. The LIA time constant and slope filter were set to $1 \mathrm{~s}$ and $12 \mathrm{~dB} /$ octave, respectively, corresponding to a detection bandwidth of $\Delta f=0.25 \mathrm{~Hz}$. A personal computer
(PC) equipped with data acquisition (DAQ) card was used to record the experimental data.

The QEPAS spectrophone consists of a custom-made QTF with a fundamental resonance frequency of $2.8 \mathrm{kHz}$ coupled with a single-tube AmR in an on-beam configuration. The two prongs of the QTF are separated by $700 \mu \mathrm{m}$, and are $17 \mathrm{~mm}$ long $(L), 1 \mathrm{~mm}$ thick $(T)$, and $0.25 \mathrm{~mm}$ wide $(W)$. Details of the QTF fabrication process are described in Ref. 28. From the QTF dimensions and geometry we estimated a fundamental resonance frequency of $2.8 \mathrm{kHz}$ and a 1st overtone resonance frequency of $17.7 \mathrm{kHz}$, according to $f_{n}=\frac{\pi T}{8 \sqrt{12} L^{2}} \sqrt{\frac{E}{\rho}} m^{2}$, where $E$ represents the quartz Young's modulus, $\rho$ its density, and $\mathrm{m}=1.194$ and $\mathrm{m}=2.988$ for the fundamental and the overtone modes, respectively. ${ }^{4,5,28} \mathrm{~A}$ function generator (Tektronix, AFG3102) was used to scan the frequency of the electrical signal applied to the custom QTF, and the LIA was used to record the QTF response at each frequency. The measured resonance frequency $f_{\mathrm{n}}, Q_{\mathrm{n}}$ value, and equivalent resistance $R_{\mathrm{n}}$ of the QTF for the fundamental mode $(\mathrm{n}=0)$ and the first overtone mode $(\mathrm{n}=1)$ are $f_{0}=2.868 \mathrm{kHz}, Q_{0}=5800, R_{0}=721 \mathrm{k} \Omega$ and $f_{1}=17.741 \mathrm{kHz}$, $Q_{1}=12553, R_{1}=162 \mathrm{k} \Omega$, respectively. The measured resonance frequencies are in good agreement with theoretical predictions. The QTF support losses were significantly reduced due to the optimization of geometrical ratio $L / T$, providing a more than $2 \times$ higher $Q$-factor for the overtone mode with respect to the fundamental mode. ${ }^{4,5} \mathrm{~A}$ single-tube AmR made of stainless steel with the inner and outer diameters of $0.62 \mathrm{~mm}$ and $0.98 \mathrm{~mm}$, respectively, was inserted between the QTF prongs, to realize a SO-QEPAS spectrophone. This selection of inner and outer diameters of the AmR is based on our previous experimental results reported
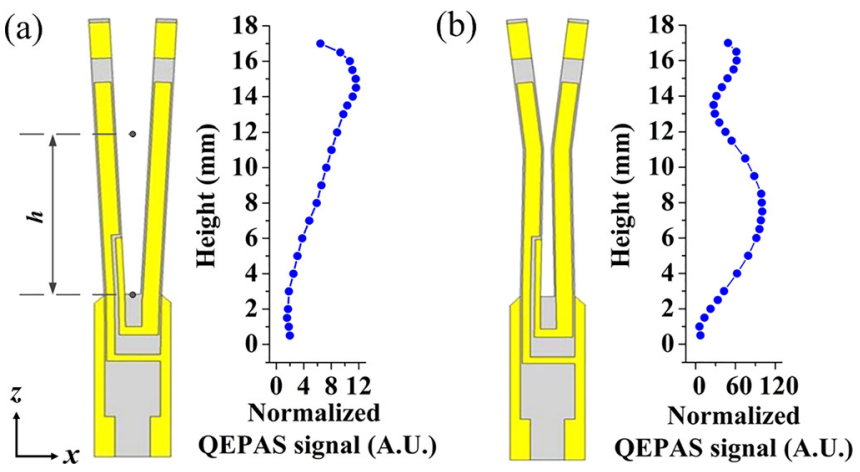

FIG. 2. QEPAS signal amplitude for fundamental (a) and overtone (b) resonance modes at different heights along the QTF $z$ axis. The QEPAS signal was normalized to the absolute atmospheric humidity. 
in Ref. 29. The waist thickness in the middle of the AmR was polished to $\sim 650 \mu \mathrm{m}$ (see Fig. 2(b)), in order to accommodate the AmR between the prongs of the QTF. Two narrow slits were symmetrically made in the middle of the AmR (see Fig. 1) to produce an acoustic diverging flow from the AmR to effectively drive the vibration of the QTF prongs. This approach avoids the splitting of the AmR into two pieces, resulting in the formation of quasi standard acoustic standing waves. ${ }^{29}$ The slit width and length were set to $\sim 90 \mu \mathrm{m}$ and $\sim 200 \mu \mathrm{m}$, respectively, since larger slit widths disperse the acoustic energy, while smaller slit sizes limit the coupling of the acoustic energy.

According to the HITRAN database,${ }^{30}$ a $\mathrm{H}_{2} \mathrm{O}$ absorption line located at $7303.23 \mathrm{~cm}^{-1}$ with the line intensity of $8.05 \times 10^{-22} \mathrm{~cm} / \mathrm{mol}$ was selected as the target absorption line for QEPAS detection. The water concentration in ambient air was monitored by means of direct absorption spectroscopy using a diode laser emitting at $7306.75 \mathrm{~cm}^{-1}$ and a photodiode detector with an effective optical absorption length of $50 \mathrm{~cm} .^{31}$ The uncertainty of water concentration measurement is $\pm 1 \%$ of the measuring mean value. The performance of the bare custom QTF without an AmR was first measured for both fundamental and 1st overtone resonance modes. The laser fiber-coupled collimator was installed on a $\mathrm{XYZ}$ translation mount to scan the laser beam focus along the QTF $z$ axis, starting from the QTF support. The optimum laser beam position was identified by measuring the $2 f$ wavelength modulation-based QEPAS amplitudes profile along QTF $z$ axis. The QEPAS signal profile normalized to the water concentrations as a function of height $(h)$ is shown in Fig. 2, for the fundamental mode (panel (a)) and the 1st overtone flexural mode (panel (b)). When operating in the fundamental mode, the maximum signal amplitude occurred at $h=15 \mathrm{~mm}$, rather than the QTF top with a maximum torque at $h=17 \mathrm{~mm}$. This is attributed to an acoustic wave leakage from the QTF top. The initial signal decrease is due to photo-thermoelastic phenomena that occur near the QTF base. ${ }^{32}$ The QEPAS signal profile obtained when the QTF operates with the 1st overtone mode shows two signal antinodes at $h=7.5 \mathrm{~mm}$ and $16 \mathrm{~mm}$, respectively. The signal amplitude at $h=7.5 \mathrm{~mm}$ is $\sim 2$ times higher than that measured at $h=16 \mathrm{~mm}$, due to partial acoustic wave leakage from the QTF top. The maximum signal amplitude obtained by the custom QTF 1st overtone resonance mode is $\sim 8$ times higher than that obtained by fundamental resonance mode and the corresponding optimum modulation depth (maximum QEPAS amplitude) for the fundamental and 1st overtone resonance mode were $0.35 \mathrm{~cm}^{-1}$ and $0.44 \mathrm{~cm}^{-1}$, respectively.

The acoustic coupling efficiency between the QTF and the AmR strongly depends on the AmR vertical position. ${ }^{29,33}$ Previous work showed that variations in AmR position with respect to the QTF $z$-axis as small as $0.1 \mathrm{~mm}$ can significantly affect the spectrophone performance in conventional QEPAS. ${ }^{33}$ A group of AmR holders of various heights was printed by a Cube Pro 3D printer, and used to position the single-tube AmRs at different heights along the QTF z-axis. For the QTF fundamental resonance frequency, the acoustic wavelength $\lambda_{\mathrm{s}}$ occurs at $120 \mathrm{~mm}$ at atmospheric pressure, while, for the QTF 1st overtone resonance mode,
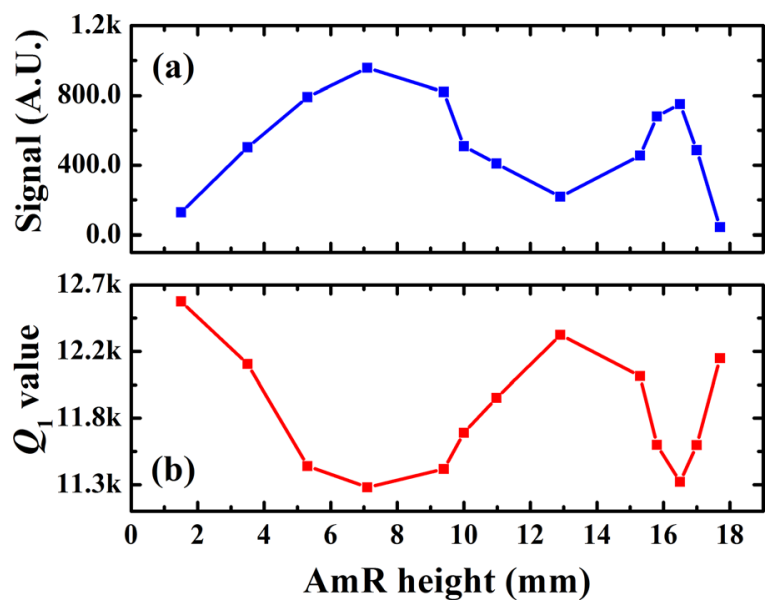

FIG. 3. SO-QEPAS signal amplitude (a) and $Q_{1}$ value (b), as a function of AmR height.

$\lambda_{\mathrm{s}}=19 \mathrm{~mm}$. Therefore, the SO-QEPAS configuration can be implemented only if operating in the 1st overtone mode and the length of single-tube AmR was set to $\sim 9.5 \mathrm{~mm}\left(\lambda_{\mathrm{s}} / 2\right)$. The $2 f$ wavelength modulation-based QEPAS signal and the corresponding QTF $Q_{1}$ values versus the AmR vertical positions were measured, and the results are shown in Fig. 3, where the signals were normalized to the $\mathrm{H}_{2} \mathrm{O}$ concentration. Accordingly to what observed with the bare QTF, the SOQEPAS signal shows two antinodes at $h=7 \mathrm{~mm}$ and $16.5 \mathrm{~mm}$. Correspondingly, the $Q$-factor value shows two valleys where the signal antinodes occur (see Fig. 3(b)), indicating the achievement of local maxima in the acoustic coupling between the QTF and the AmR. The lowest $Q_{1}$ value measured in air is 11294 at $h=7 \mathrm{~mm}$, and is lower than that measured for the bare QTF (12553). This indicates an improved acoustic coupling between the QTF and AmR. As a result, the SO-QEPAS spectrophone with an AmR mounting at a height of $7 \mathrm{~mm}$ gives the highest signal amplitude, corresponding to a signal enhancement factor of $\sim 10$, compared with a bare custom QTF.

The next step was the optimization of the single-tube AmR length, since the optimum values occur between $\lambda_{s} / 2$ and $\lambda_{s}{ }^{25,29}$ With the AmR vertical position at $h=7 \mathrm{~mm}$, the AmR lengths were changed from $19 \mathrm{~mm}\left(\sim \lambda_{s}\right)$ to $9.5 \mathrm{~mm}$

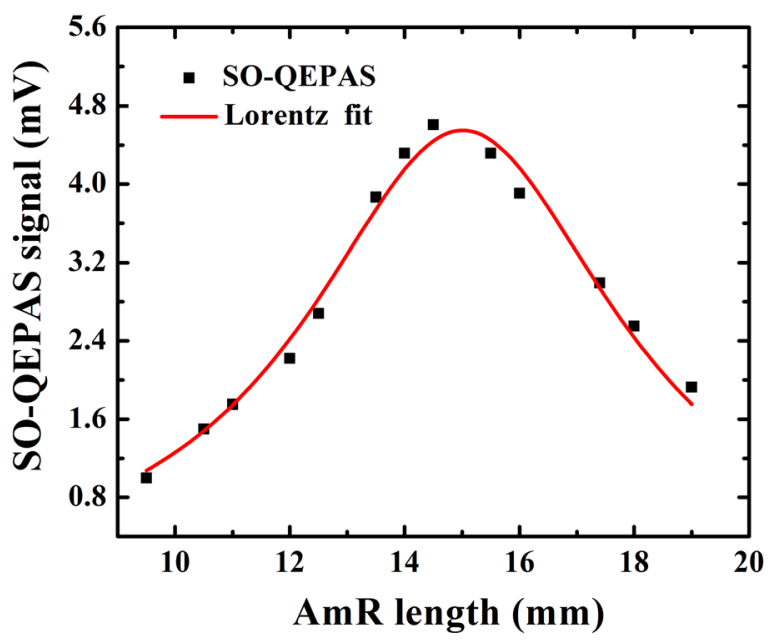

FIG. 4. SO-QEPAS signals as a function of AmR lengths. 


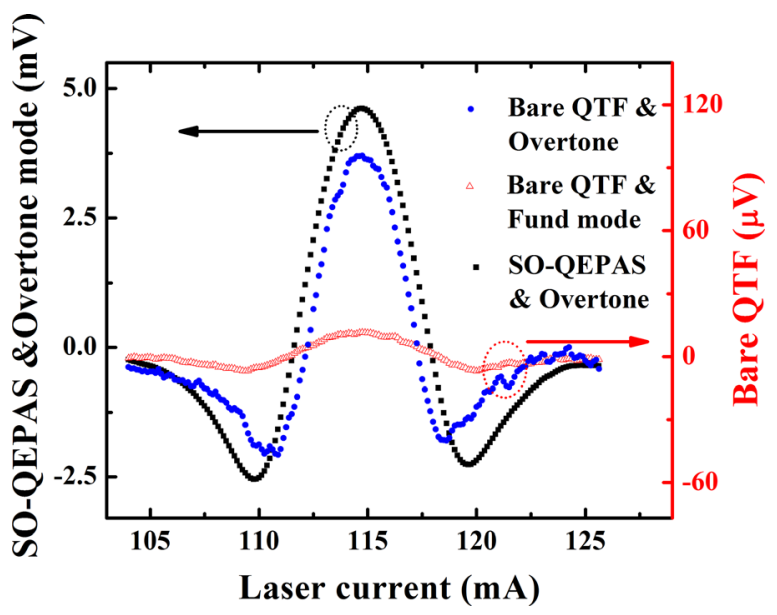

FIG. 5. $2 f$ signals obtained by overtone resonance enhanced SO-QEPAS spectrophone and bare custom QTF operating in the fundamental and 1st overtone resonance modes.

$\left(\sim \lambda_{s} / 2\right)$. Figure 4 depicts the measured SO-QEPAS signals versus the AmR lengths. The signals amplitudes were normalized to $1 \%$ water concentration and the data points were fitted by a Lorentz line shape. The maximum signal amplitude was obtained for a single-tube length of $14.5 \mathrm{~mm}$. The highest signal amplitude obtained is $4.6 \mathrm{mV}, \sim 5$ times larger than that obtained with an AmR length of $\lambda_{s} / 2(9.5 \mathrm{~mm})$ and $\sim 50$ times larger than that measured using a bare QTF operating in the 1 st overtone resonance mode. The observation of an optimal tube length $>\lambda_{s} / 2$ is a clear evidence that the $1 \mathrm{st}$ harmonic acoustic standing waves in the AmR were partially distorted by the two slits present in the resonator. ${ }^{29}$

A comparison of the $2 f$ signals obtained with the SOQEPAS spectrophone operating in the QTF 1st overtone resonance mode and with the bare custom QTF operating in its fundamental and 1 st overtone resonance modes are displayed in Fig. 5. The overtone resonance enhanced SOQEPAS spectrophone reached the peak signal amplitude of $4.6 \mathrm{mV}$, which is $\sim 380$ times higher than that obtained with the bare QTF operating in fundamental resonance mode. The noise of the QEPAS spectrophone was measured by tuning the laser wavelength away from the $\mathrm{H}_{2} \mathrm{O}$ absorption line. The noise level $(1 \sigma)$ for the overtone resonance enhanced SO-QEPAS spectrophone and the bare QTF were $2 \mu \mathrm{V}$ and $1.6 \mu \mathrm{V}$, respectively, which are slightly higher than the calculated theoretical thermal noise of $1.6 \mu \mathrm{V}$ and $0.7 \mu \mathrm{V}$. For the overtone resonance enhanced SO-QEPAS spectrophone, a signal-to-noise ratio (SNR) of 2300 was obtained with a laser power of $23 \mathrm{~mW}$, corresponding to a normalized noise equivalent absorption coefficient (NNEA) of $2.76 \times 10^{-9} \mathrm{~cm}^{-1} \mathrm{~W} / \sqrt{\mathrm{Hz}}$.

In conclusion, an overtone resonance enhanced SOQEPAS spectrophone was developed based on a custommade $2.8 \mathrm{kHz}$ QTF with large prong spacing, properly designed to provide an enhanced QEPAS signal for the overtone resonance mode with respect to the fundamental one. Due to the high overtone resonance frequency, the optimal AmR length was $14.5 \mathrm{~mm}$, only $5 \mathrm{~mm}$ longer than that employed so far with a commercial $32 \mathrm{kHz}$ QTF. The combination of the overtone enhancement and SO-QEPAS techniques yielded a sensitivity enhancement factor of $\sim 380$, with respect to the bare custom QTF operated in fundamental resonance mode. The sensitivity enhancement factor is $\sim 13$ times higher than that attained by a conventional QEPAS spectrophone based on commercial $32 \mathrm{kHz} \mathrm{QTF}^{25}$ The experimental setups for commercial and custom QTFs are the same. The commercial QTF can be replaced with a custom QTF in QEPAS sensors. Moreover, the large prong spacing of the custom QTF facilitates optical alignment of the laser beam through the spectrophone and extends the QEPAS applications. Further improvement of the detection sensitivity can be expected by assembling two acoustic resonators at the two antinodes $(h=7 \mathrm{~mm}$ and $16.5 \mathrm{~mm})$ along QTF prong to increase the energy accumulation in the spectrophone. Furthermore, since the first flexural overtone mode is characterized by two nodal points of motion of the prongs, an optimal charge collection requires polarity changes of the electrodes along the prongs, which can be realized by an octupole gold pattern configuration. Thus, an additional enhancement of the 1st overtone QEPAS SNR is expected if employing this type of QTF contact design.

Lei Dong acknowledges support by the National Natural Science Foundation of China (Grant Nos. 61622503, 61575113, and 61275213). Frank Tittel acknowledges support by the National Science Foundation (NSF) ERC MIRTHE award and the Robert Welch Foundation (Grant No. C-0586). The authors from Dipartimento Interateneo di Fisica di Bari acknowledge financial support from two Italian research Projects Nos. PON02 00675 and PON02 00576 .

${ }^{1}$ A. A. Kosterev, Y. A. Bakhirkin, R. F. Curl, and F. K. Tittel, Opt. Lett. 27, 1902 (2002).

${ }^{2}$ K. Liu, X. Guo, H. Yi, W. Chen, W. Zhang, and X. Gao, Opt. Lett. 34, 1594 (2009).

${ }^{3}$ P. Patimisco, G. Scamarcio, F. K. Tittel, and V. Spagnolo, Sensors 14, 6165 (2014).

${ }^{4}$ F. K. Tittel, A. Sampaolo, P. Patimisco, L. Dong, A. Geras, T. Starecki, and V. Spagnolo, Opt. Express 24, A682 (2016).

${ }^{5}$ A. Sampaolo, P. Patimisco, L. Dong, A. Geras, G. Scamarcio, T. Starecki, F. K. Tittel, and V. Spagnolo, Appl. Phys. Lett. 107, 231102 (2015).

${ }^{6}$ L. Dong, H. Wu, H. Zheng, Y. Liu, X. Liu, W. Jiang, L. Zhang, W. Ma, W. Ren, W. Yin, S. Jia, and F. K. Tittel, Opt. Lett. 39, 2479 (2014).

${ }^{7}$ Y. Ma, Y. He, X. Yu, C. Chen, R. Sun, and F. K. Tittel, Sens. Actuators, B 233, 388 (2016).

${ }^{8}$ Z. Wang, Z. Li, and W. Ren, Opt. Express 24, 4143 (2016).

${ }^{9}$ J. P. Waclawek, H. Moser, and B. Lendl, Opt. Express 24, 6559 (2016).

${ }^{10}$ H. Wu, A. Sampaolo, L. Dong, P. Patimisco, X. Liu, H. Zheng, X. Yin, W. Ma, L. Zhang, W. Yin, V. Spagnolo, S. Jia, and F. K. Tittel, Appl. Phys. Lett. 107, 111104 (2015).

${ }^{11}$ S. Borri, P. Patimisco, A. Sampaolo, H. E. Beere, D. A. Ritchie, M. S. Vitiello, G. Scamarcio, and V. Spagnolo, Appl. Phys. Lett. 103, 021105 (2013).

${ }^{12}$ H. Zheng, L. Dong, X. Yin, X. Liu, H. Wu, L. Zhang, W. Ma, W. Yin, and S. Jia, Sens. Actuators, B 208, 173 (2015).

${ }^{13}$ M. Jahjah, W. Jiang, N. P. Sanchez, W. Ren, P. Patimisco, V. Spagnolo, S. C. Herndon, R. J. Griffin, and F. K. Tittel, Opt. Lett. 39, 957 (2014).

${ }^{14}$ K. Liu, W. Zhao, L. Wang, T. Tan, G. Wang, W. Zhang, X. Gao, and W. Chen, Opt. Commun. 340, 126 (2015).

${ }^{15}$ H. Yi, R. Maamary, X. Gao, M. W. Sigrist, E. Fertein, and W. Chen, Appl. Phys. Lett. 106, 101109 (2015).

${ }^{16}$ Y. Cao, N. P. Sanchez, W. Jiang, R. Griffin, F. Xie, L. C. Hughes, C. Zah, and F. K. Tittel, Opt. Express 23, 2121 (2015).

${ }^{17}$ W. Ren, W. Jiang, N. P. Sanchez, P. Patimisco, V. Spagnolo, C. Zah, F. Xie, L. C. Hughes, R. J. Griffin, and F. K. Tittel, Appl. Phys. Lett. 104, 041117 (2014). 
${ }^{18}$ L. Dong, V. Spagnolo, R. Lewicki, and F. K. Tittel, Opt. Express 19, 24037 (2011).

${ }^{19}$ T. N. Ba, M. Triki, G. Desbrosses, and A. Vicet, Rev. Sci. Instrum. 86, 023111 (2015).

${ }^{20}$ G. Wysocki, A. A. Kosterev, and F. K. Tittel, Appl. Phys. B 85, 301 (2006).

${ }^{21}$ S. Böttger, M. Köhring, U. Willer, and W. Schade, Appl. Phys. B 113, 227 (2013).

${ }^{22}$ A. Sampaolo, P. Patimisco, M. Giglio, L. Chieco, G. Scamarcio, F. K. Tittel, and V. Spagnolo, Opt. Express 24, 15872 (2016).

${ }^{23}$ H. Zheng, L. Dong, Y. Ma, H. Wu, X. Liu, X. Yin, L. Zhang, W. Ma, W. Yin, L. Xiao, and S. Jia, Opt. Express 24, A752 (2016).

${ }^{24}$ Y. Ma, Y. He, X. Yu, J. Zhang, R. Sun, and F. K. Tittel, Appl. Phys. Lett. 108, 091115 (2016).

${ }^{25}$ L. Dong, A. A. Kosterev, D. Thomazy, and F. K. Tittel, Appl. Phys. B 100, 627 (2010).
${ }^{26}$ V. Spagnolo, L. Dong, A. A. Kosterev, D. Thomazy, J. H. Doty III, and F. K. Tittel, Appl. Phys. B 103, 735 (2011).

${ }^{27}$ V. Spagnolo, P. Patimisco, R. Pennetta, A. Sampaolo, G. Scamarcio, M. S. Vitiello, and F. K. Tittel, Opt. Express 23, 7574 (2015).

${ }^{28}$ P. Patimisco, A. Sampaolo, L. Dong, M. Giglio, G. Scamarcio, F. K. Tittel, and V. Spagnolo, Sens. Actuators, B 227, 539 (2016).

${ }^{29}$ H. Zheng, L. Dong, A. Sampaolo, H. Wu, P. Patimisco, X. Yin, W. Ma, L. Zhang, W. Yin, S. Jia, and F. K. Tittel, Opt. Lett. 41, 978 (2016).

${ }^{30}$ See http://www.hitran.com for absorption line information.

${ }^{31}$ X. Yin, L. Dong, H. Zheng, X. Liu, H. Wu, Y. Yang, W. Ma, L. Zhang, W. Yin, L. Xiao, and S. Jia, Sensors 16, 162 (2016).

${ }^{32}$ M. Spajer, B. Cavallier, S. Euphrasie, G. Matten, X. Vacheret, P. Vairac, D. Vernier, and A. Jalocha, Appl. Phys. Lett. 103, 201111 (2013).

${ }^{33}$ H. Wu, L. Dong, W. Ren, W. Yin, W. Ma, L. Zhang, S. Jia, and F. K. Tittel, Sens. Actuators, B 206, 364 (2015). 